\title{
Vigilância das Doenças Crônicas e Ocupacionais: Como Passar das Propostas às Ações?
}

Heleno Rodrigues Corrêa Filho*

O texto foi produzido sob o tema - "os idosos de 2020 serão os trabalhadores dos anos 90", onde se discute o papel da Epidemiologia como meio auxiliar na distribuição de cuidados que promovam os principais objetivos das ciências aplicadas à saúde: - aliviar a dor e prolongar a vida. Tais objetivos são encarados como metas submetidas aos critérios tradicionais de escolher prioridades para atenção à saúde - a transcendência, vulnerabilidade e a magnitude dos danos impostos pelos agravos ocupacionais e não ocupacionais do tipo crônico-degenerativo. A população idosa é especialmente considerada pelo acúmulo de condições desvantajosas em cenário onde os que estão em idade produtiva já não contam com proteção. Para obter-se a eqüidade no direito à saúde são enfatizadas a formação continuada de recursos humanos e a incorporação tecnológica de recursos gerenciais como meios para superar os modelos de desenvolvimento social baseados na exclusão da maioria para a valorização do consumo e maximização do lucro.

No painel realizado pela Associação Paulista de Saúde Pública foram chamados dois especialistas para expor seus pontos de vista sobre a contribuição esperada a partir da epidemiologia para o controle dos agravos ocupacionais e crônicos e degenerativos. $\mathrm{O}$ conhecimento prévio do tema e da disposição dos painelistas serviu para desenvolver a temática aqui abordada, com a finalidade de estimular o debate realizado com os participantes do encontro.*

A descrição da magnitude dos problemas crônicos é acompanhada da descrição do aumento da proporção de idosos na população geral e na recusa dos

* Professor Assistente do Departamento de Medicina Preventiva da UNICAMP

* Prof. Dr. Ricardo C.Cordeiro - UNESP/FCM-Botucatu-Dep.Saúde Pública e Prof. Dr. Luiz Roberto Ramos-UNEFESP/EPM. 
formuladores das políticas de saúde em reconhecer este fato. São associados ao mesmo a mudança nos perfis de morbimortalidade, requerendo providências preventivas para as quais o conhecimento vigente ou é ineficaz ou ineficiente, associando altos custos operacionais que comprometem também a efetividade.

Em relação aos idosos, o sistema médico-hospitalar consegue mais a realização do lucro que paga os equipamentos e a tecnologia do que a difusão da assistência através de um sistema sanitário amplamente acessível à população carente.

A perda da autonomia, conseqüente à limitação e seqüela de doenças vasculares, osteo-musculares e as várias formas de demência, exigem dos epidemiologistas a disponibilidade de informações detalhadas por faixas etárias diferentes das tradicionalmente apresentadas em estatísticas oficiais. As informações sobre doenças com "50 e mais" anos, tornam impossível a discriminação das necessidades de intervenção em idades onde um ano a mais de vida significa alta complexidade adicional em cuidados e dimensionamento de recursos ${ }^{(9)}$.

A inexistência virtual de apoio não hospitalar para a chamada terceira idade (mais de 70 anos), cria em relação aos idosos a obrigação de permanecer sem ajuda, até que a gravidade de seus problemas os tenha levado para um atendimento terminal e sem resolutividade ${ }^{(3)}$.

Outro fator, que liga as duas temáticas do painel, é a exclusão de pessoas que cuidam dos idosos do mercado de trabalho formal e mesmo da atividade econômica. Estima-se que para cada idoso doente sem autonomia seja excluída pelo menos uma pessoa que deve acompanhá-lo em sua dependência para cuidados básicos de sobrevivência ${ }^{(9)}$.

Os que afortunadamente estão incluídos no mercado de trabalho em idade produtiva, são clientes potenciais de um sistema de saúde onde a epidemiologia não está aplicada com seu potencial analítico. Também para estes a lógica presentemente aplicada é a da exclusão: tanto a exclusão do consumo quanto dos direitos de proteção específica e promoção da saúde.

São características do grupo dos trabalhadores a subnotificação ou a simples inexistência de sistemas de vigilância de seus agravos, em que pese a tendência recente de desenvolvimento de sistemas públicos especializados em cidades do estado de São Paulo.

As deficiências dos profissionais de saúde para conhecer e enfrentar os problemas de saúde do trabalhador são de formação técnica insuficiente, ausência de políticas públicas específicas e a predominância do modelo empresarial de atendimento médico exclusivo fora da saúde pública (5).

A este panorama agrega-se a inexistência de informações epidemiológicas específicas, diluindo os dados sobre acidentes, doenças e atenção médica em múltiplas instituições com bases de dados não interligadas. 
Os órgãos de vigilância à saúde não dispõem de informações epidemiológicas validadas para trabalhadores brasileiros sobre os níveis aceitáveis de riscos e exposição a agentes agressivos com base em Limites de Tolerância Biológica (LTB's) estudados em condições reais de nutrição, esforço físico e suscetibilidade individual que permitam o acompanhamento de higienistas industriais e profissionais de saúde ${ }^{(6)}$. Abre-se neste particular um grande espaço para a atuação dos programas públicos de Saúde do Trabalhador (PST's), como terrenos propícios para estudos epidemiológicos descritivos, analíticos (especialmente do tipo caso-controle) e mesmo de intervenção ${ }^{(5)}$.

\section{Temática sob discussão}

Com a contribuição dos painelistas, e com dados epidemiológicos disponíveis, pôde-se afirmar que na perspectiva atual, os trabalhadores desassistidos da década de 90 serão os idosos da década de 2020 .

Já no período atual, a formulação das políticas públicas desfavorece o trabalho epidemiológico de localizar quais cuidados médico-sanitários seriam prioritários para ambos os grupos.

A magnitude dos danos epidemiológicos de grupos de trabalhadores e idosos depende de sistemas de vigilância epidemiológica integrados, permeando tanto a assistência médico-sanitária pública quanto privada. $\mathrm{O}$ desconhecimento relativo a quem atende o quê pode ser superado com a integração local e regional de dados e com o aumento da autonomia dos municípios na gestão dos sistemas de saúde.

A transcendência dos problemas, seria um fator a ser necessariamente conhecido. Na população idosa, as doenças com incapacitação maior e maior custo de atenção deveriam ser alvo de coleta de dados epidemiológicos detalhados por idade e descritores socio-econômicos específicos. A demência é especialmente dramática por comprometer pessoas sem a existência de espaço mínimo para a assistência aos necessitados, além da incompreensão social do significado da dependência por problemas mentais.

$\mathrm{Na}$ saúde do trabalhador, o principal obstáculo para o conhecimento de problemas transcendentes seria a unificação das bases de dados que permitam localizar problemas que os exclua do trabalho ou que impossibilite a fruição plena de sua vida produtiva.

A vulnerabilidade dos problemas de saúde enfrentados por trabalhadores e idosos está vinculada ao manejo eficiente da tecnologia disponível. A epidemiologia assume aí papel destacado na avaliação da eficiência e efetividade dos métodos e técnicas empregados. A efetividade é especialmente importante por ser determinada pela realidade local, subordinada aos contextos social, cultural e político. 
Os estudos epidemiológicos sobre efetividade necessitam de sistemas de informação locais informatizados, com gerenciamento de complexidade gradativa e hierarquizada de maior autonomia local. Uma estratégia mais eficiente para não superlotar técnicos do nível local e regional seria o estabelecimento de padrões para informação epidemiológica em doenças diferentes, a serem aplicados de modo seletivo, intermitente e/ou rotativo, segundo as necessidades locais de saúde. A padronização de rotinas assistenciais clínicas mínimas deveria ser estudada com critérios epidemiológicos e difundida através dos sistemas de atenção à saúde, indicando para cada nível assistencial quais as rotinas mais adequadas para produzir o melhor efeito com os recursos disponíveis.

Ainda na área relativa à vulnerabilidade, os estudos de eficiência epidemiológica das técnicas assistenciais têm lugar, aliando as técnicas de gerenciamento para a obtenção de informações sobre atributos das metodologias assistenciais a saber - onde, quando e quanto se aplica a cada tecnologia empregada. Estes estudos, praticamente inexistentes em nosso meio, são necessários para a discussão democrática na alocação de recursos orçamentários e técnicos quando estiverem envolvidos usuários e profissionais, especialmente no nível local e nos conselhos comunitários de saúde.

O principal obstáculo presente hoje para a implementação de estudos epidemiológicos no campo da saúde do trabalhador e dos idosos é a política denominada de Neo-liberal. Esta política, recuperando o liberalismo estrito que antecedeu as propostas de estados com políticas públicas voltadas para o bemestar social no após-guerra (1945), tem sido implementadas, em particular no Brasil, com características perversas que impossibilitam o uso das técnicas epidemiológicas, fragmentando as fontes de informação e decisão para fundamentar políticas de saúde igualitárias. As características predominantes da política neo-liberal recente seriam:

- Retirar os cuidados de saúde coletiva e seus profissionais do mercado de trabalho, extinguindo os postos de trabalho e as definições de competência como carreiras, normas de trabalho e salários*

- Retirar o mercado dos serviços privados de saúde do controle do sistema público e das políticas de saúde coletiva, impedindo a organização de sistemas de informação epidemiológica unificados e abrangentes;

\footnotetext{
* Ler a este respeito a referência de Cordeiro, 1995-b sobre a gestão José Aristodemo Pinotti à frente da Secretaria de Estado da Saúde do Estado de São Paulo em relação à carreira de médico- sanitarista.

A este respeito: Prof. Antonio Ribeiro Franco (USP/FM-Ribeirão Preto) manifestou seu desencanto por não encontrar nos debates os militantes da saúde pública que conheceu ao longo dos anos indicando o sintoma de derrota das propsotas de criar sistemas epidemiológicos públkicos de saúde. O Dr. Marcos Drumond (Pref.Município SP) referiu que os novos sanitaristas também não estavam no debate apesar de implementar na prefeitura de SP a busca de casos atendidos em serviços de saúde com características suspeitas de acidentes do trabalho sem notificação. Outro especialista presente comentou o esforço do CVE-Centro de Vigilância Epidemiológica da Secretaria de Estado da Saúde de SP em criar um indicador epidemiológico para incapacidades.
} 
- Criar sistemas de decisão e controle independentes e fragmentários para administrar e orçamentar de modo diferente a saúde dos trabalhadores e dos idosos, dificultando ou impedindo o controle público através do sistema oficial de saúde. Cada um no seu canto e sem notícias do outro.

- O abandono das políticas de autonomia científica e tecnológicas, diminuindo ou dificultando a formação de recursos humanos com conhecimento epidemiológico especializado e impedindo a geração de novas tecnologias assistenciais eficazes e adequadas*

A característica mais perversa deste sistema baseado na exclusão é que as vítimas passam para o limbo, deixando de existir, na medida em que ao serem portadoras de um agravo ou deficiência não há mais meios epidemiológicos de reconstruir sua trajetória individual através do sistema de informações ${ }^{(4)}$.

Os atendidos no sistema de saúde, enquanto portadores de problemas, contribuem para maximizar o lucro concentrado em poucas unidades hospitalares de alto custo, inacessíveis para a grande maioria, mas aparentemente capazes de sustentar o consumo de tecnologias produzidas em nosso meio.

\section{Estratégias de enfrentamento dos obstáculos para aplícação do conhecimento epidemiológico}

A capacitação de recursos humanos em saúde e a difusão de meios padronizados de registrar e analisar a informação epidemiológica foi apontada como a forma de superar as dificuldades do modelo excludente de desenvolvimento que tenta se fazer hegemônico.

As qualidades necessárias para implementar políticas públicas de saúde com qualidades técnicas baseadas no emprego da epidemiologia dependem da qualificação da mão-de-obra atuante na assistência médico-sanitária.

O objetivo principal seria utilizar dados visando melhorar a qualidade de vida, promover e proteger a saúde, com base na análise dos dados sobre os que já contraíram os problemas e no conhecimento correto dos denominadores representando as populações sob risco.

Algumas medidas práticas foram apontadas, como: a reafirmação das políticas públicas específicas para trabalhadores em idade produtiva ${ }^{(1,11,10)} \mathrm{e}$ idosos a obrigatoriedade de emitir Comunicações de Acidentes do Trabalho inclusive para os trabalhadores do mercado informal; a definição de critérios diagnósticos para os "casos" de doenças ocupacionais ${ }^{(1)}$ e degenerativas específicas e a redefinição epidemiológica dos limites de tolerância biológica e ambientais.

* Adequação é tomada aqui como atributo que reune a eficiência e a efetividade - proposta para ser submetida a discussão. 
Em relação à assistência médico-sanitária foram ressaltados recursos já disponíveis e relevantes desde que convenientemente utilizados como: a informatização dos prontuários em serviços públicos e nas redes primária e hospitalar*; a hierarquização da rede de inteligência epidemiológica permitindo análises diretamente no nível local com maior complexidade de atributos do que no nível central ${ }^{(7)}$; a implantação de núcleos de vigilância epidemiológica em hospitais e outras providências dependentes da formação de recursos humanos especializados em epidemiologia ${ }^{(2)}$.

\section{Referências Bibliográficas}

1. ALESSI, N.P.; SILVA, G.B.; PINHEIRO, S.A.; SCOPINHO, R.A.. Formação de recursos humanos para a área de saúde do trabalhador: 0 processo de implantação de "ficha epidemiológica de saúde e trabalho" na prática dos profissionais de um centro de saúde escola. Rev.Bras. Saúde Ocup., 19(72):45-58, 1991.

2. AUGUSTO, L.G.S.; ROCHA, L.E.; FREITAS, C.U.; LACAZ, F.A.C.; BICHIR, A.. Vigilância epidemiológica de doenças ocupacionais. Rev.Bras. Saúde Ocup., 14,(54):32-64, 1986.

3. CAMPOS, F.E..Resolutividade: uma aproximação à avaliação dos serviços de saúde. Rio de Janeiro, 1988. [Tese de Doutorado - Fundação Oswaldo Cruz - Escola Nacional de Saúde Pública].

4. COHN, A.; HIRANO, S.; KARSCH, U.S.; SATO, A.K.. Acidentes do trabalho: uma forma de violência. São Paulo, Brasiliense, 1985.

5. CORDEIRO, R.. Problemas em saúde do trabalhador: perspectivas epidemiológicas. São Paulo: 1995. Comunicação Oral - (I Encontro Paulista de Epidemiologia - Associação Paulista de Saúde Pública - São Paulo, SP).

6. CORDEIRO, R..Quando começa o saturnismo?. Campinas, 1995. [Tese de Doutorado - Saúde Coletiva - UNICAMP - Faculdade de Ciências Médicas].

7. CORRÊA-FILHO, H.R. Percepção de riscos na ocupação precedendo lesões do trabalho: um estudo no município de Campinas, São Paulo, 19921993. São Paulo, 1994. [Tese de Doutorado - Faculdade de Saúde Pública da USP].

\footnotetext{
* A este respeito: a Dra. Fátima (Sec.Saúde Município de S.José dos Campos-SP) relatou caso curioso de denúncia de médicos ao Conselho Regional de Medicina de SP contra a implantação de prontuários informatizados alegando quebra do sigilo médico, como se prontuários não informatizados não implicassem em manuseio igualmente obrigado ao segredo ético. Outros profissionais resslataram a utilidade e oportunidade de implantar os sistemas denominados SIM/SINA/SINASC do Ministério da Saúde, acrescidos de atributos de identificação local de endereços hoje inexistentes nos mesmos.
} 
8. MENDES, E.V. O planejamento local da vigilância da saúde no distrito sanitário. In: OPAS/OMS - Planejamento e programação local da vigilância da saúde no distrito sanitário, Brasília, 1994, p.7-18 (Série Desenvolvimento de serviços de saúde no 13).

9. RAMOS, L.R. Doenças crônicas e degenerativas: perspectivas epidemiológicas. São Paulo, 1995. Comunicação Oral - (I Encontro Paulista de Epidemiologia - Associação Paulista de Saúde Pública - São Paulo, SP).

10. SANTOS, U.P.; WÜNCH FILHO, V.; CARMO, J.C.; SETTIMI, M.M.; URQUIZA, S.D.; HENRIQUES, C.M.P.. Sistema de vigilância epidemiológica para acidentes do trabalho: experiência na zona norte do município de São Paulo (Brasil). Rev. Saúde Pública, 24: 286-93, 1990.

11. SANTOS, U.P.; SETTIMI, M.M.; WÜNSCH FILHO, V.; CARMO, J.C.. A questão dos acidentes de trabalho. In: Costa, D.F.; Carmo, J.C.; Setimmi, M.M.; Santos, U.P.. Programa de saúde dos trabalhadores: a experiência da Zona Norte: uma alternativa em saúde pública. São Paulo, HUCITEC, 1989 p.157-78. 